\title{
Altered Function of Ventrolateral Prefrontal Cortex in Adolescents with Peer Verbal Abuse History
}

\author{
Sang Won Lee, ${ }^{1,2}$, Jeewook Choi ${ }^{3}$, Jong-Sun Lee ${ }^{4}$, Jae Hyun Yoo', Ko Woon Kim¹, \\ Dongchan $\mathrm{Kim}^{5}$, HyunWook Park ${ }^{5}$, and Bumseok Jeong ${ }^{1,6} \bowtie$ \\ ${ }^{1}$ Computational Affective Neuroscience and Development Laboratory, Graduate School of Medical Science and Engineering, KAIST, \\ Daejeon, Republic of Korea \\ ${ }^{2}$ Department of Psychiatry, Kyungpook National University School of Medicine, Daegu, Republic of Korea \\ ${ }^{3}$ Department of Psychiatry, Daejeon St. Mary's Hospital, The Catholic University of Korea College of Medicine, Daejeon, Republic of Korea \\ ${ }^{4}$ Department of Psychology, Kangwon National University, Chuncheon, Republic of Korea \\ ${ }^{5}$ Department of Electrical Engineering, KAIST, Daejeon, Republic of Korea \\ ${ }^{6} \mathrm{KI}$ for Health Science and Technology, KAIST Institute, KAIST, Daejeon, Republic of Korea
}

Objective Previous studies showing the association of exposure to peer (PeVA) and parental verbal abuse in childhood with structural alterations in the young adult brain suggest functional changes in adolescence. In this functional MRI study, we investigated the effects of exposure to PeVA, during elementary and middle school periods, on brain response to emotional words, in high school students.

Methods An emotional Stroop task consisting of swear, negative, positive, and neutral words was performed during functional MRI scan for 23 subjects who were divided into low- and high exposure groups to PeVA.

Results High-PeVA group had a higher depression score, greater left ventrolateral prefrontal cortex (VLPFC) activity, and higher left VLPFC-left hippocampus connectivity in swear word conditions. The VLPFC activity and left VLPFC-left hippocampus connectivity was negatively related to the severity of anxiety and depressive symptoms, respectively.

Conclusion These preliminary findings support the hypothesis that exposure to PeVA, during childhood, is an aversive stimulus associated with meaningful functional change in emotional regulation network, showing hypersensitivity to swear words, at middle adolescence.

Psychiatry Investig 2017;14(4):441-451

Key Words Verbal abuse, Emotional trauma, Ventrolateral prefrontal cortex, Adolescence, Depression.

\section{INTRODUCTION}

Childhood maltreatment is closely linked to increased vulnerability to behavioral problems related to self-control, such as impulsivity, substance abuse, and depression. ${ }^{1}$ Among various childhood adversities, the harmful effects of parental verbal abuse (PaVA) and peer verbal abuse (PeVA) are comparable to other forms of abuse., ${ }^{2,3}$ In addition, maltreatment during early adolescence has significant negative effects on the brain development, thus it can leave severe scars. ${ }^{4,5}$

Although verbal abuse creates detrimental effects, adoles-

Received: January 25, 2016 Revised: May 11, 2016

Accepted: June 30, 2016 Available online: May 16, 2017

$\triangle$ Correspondence: Bumseok Jeong, MD, PhD

Graduate School of Medical Science and Engineering, Korea Advanced Institute of Science and Technology (KAIST), 291 Daehak-ro, Yuseong-gu, Daejeon 34141, Republic of Korea

Tel: +82-42-350-4245, Fax: +82-42-350-7160, E-mail: bs.jeong@kaist.ac.kr

(c) This is an Open Access article distributed under the terms of the Creative Commons Attribution Non-Commercial License (http://creativecommons.org/licenses/by$\mathrm{nc} / 4.0$ ) which permits unrestricted non-commercial use, distribution, and reproduction in any medium, provided the original work is properly cited. cents seems to use swear words without awareness of its seriousness. Approximately two out of three Korean middle and high school students use swear words more than once a day without recognizing the exact meaning. ${ }^{6}$ In the United States, about one out of three adolescents in grade 6-10 have suffered from verbal bullying; ${ }^{7}$ male and middle school aged students were more frequently involved. ${ }^{8}$ Therefore, while children are exposed to swearing through their everyday lives, especially in school, swear words are considered as a normal form of communication among them. Although there is much evidence of the negative effect of hurtful words, ${ }^{2,3}$ it is still unclear how exposure to swear words, mostly not intended to hurt someone, is detrimental to the brains of children with various degrees of past PeVA experiences. That may be one reason that parents have not easily answered the adolescent's question: "Why we should not use swear words?"

In neuroimaging studies with victims of childhood abuse, the disruption of the frontolimbic network (FLN) has been a frequently reported problem, ${ }^{1}$ and this problem seems to be re- 
lated to mood dysregulation. ${ }^{9,10}$ In diffusion tensor imaging (DTI) studies, the main fiber tracts connecting the prefrontal cortex and subcortical regions were damaged by emotional abuse, ${ }^{11,12}$ and the disturbance in cingulum bundle was related to depressive symptoms. ${ }^{12}$ In addition, a change in the functional connectivity between the ventromedial prefrontal cortex (VMPFC) and amygdala that is related to early life stress is related with adolescent depression and anxiety. ${ }^{13}$ Within the FLN, the ventrolateral prefrontal cortex (VLPFC) can also be impaired by abuse experiences. ${ }^{14}$ The VLPFC was engaged in evaluation and regulation of emotion ${ }^{15,16}$ and also involved in processing the negative life experiences-related information. ${ }^{17-20}$ In previous brain imaging studies using emotional Stroop task, while the activities of the anterior cingulate cortex (ACC), the amygdala, and dorsolateral prefrontal cortex (DLPFC) were frequently reported in healthy participants, ${ }^{21-23}$ the VLPFC was activated by panic-related words in panic disorder patients ${ }^{17}$ and combat-related words in post-traumatic stress disorder (PTSD) patients. ${ }^{18}$ Also, retrieval of negative autobiographical experiences also can trigger the activity in the VLPFC. ${ }^{20}$ Previous study showing anatomical attenuation of prefrontal cortex in subjects with childhood sexual abuse at ages 14-16 years ${ }^{5}$ suggested that prefrontal function maybe altered in middle adolescence with past PeVA. Thus, we hypothesized that PeVA experiences during childhood or middle school life can modulate the brain activity, especially in the VLPFC, response to swear words and the functional change are associated with greater psychiatric symptoms at middle adolescence.

To verify our hypotheses, an emotional Stroop paradigm was developed to induce an implicit emotional processing to swear words. During this task, functional magnetic resonance imaging (fMRI) was performed to measure the brain activities, especially focused on the VLPFC, amygdala, and hippocampus and the interaction among these regions.

Our study had three main purposes: 1) ascertain the effects of the implicit exposure to swear words, compared with other emotional effects, on brain function, 2) explain how previous PeVA modulates brain activities and the functional connectivity within the FLN, focused on the VLPFC, during the implicit processing of emotional words, especially swear words, 3) explore the relatedness between these changes and maladaptive behaviors. This study might provide the background needed to understand the effects of swearing on brain networks and modulatory impacts of previous PeVA experiences.

\section{METHODS}

\section{Subjects}

To recruit subjects that would represent the distribution of peer verbal abuse (PeVA) in the general student population, we first introduced an investigation of language use in Korean adolescents. In our study, only male students were recruited since the prevalence of verbal bullying is higher males than in females ${ }^{7,8}$ and abusive experiences have different effects on brain development according to sex. ${ }^{24,25}$ We considered that including sex effects on a small sample can reduce statistical power in $\mathrm{fMRI}$ analysis. In addition, we enrolled only first year high school students who were just after sensitive periods to various emotional abuses. Previous studies have reported that age 12-13 was a peak one of PeVA exposure, ${ }^{3}$ and several studies suggest that the early adolescence is a critical period for brain development including FLN. ${ }^{4,26,27}$ Furthermore, highly competitive academic environment might be a significant confounding factor in Korean high school students. Therefore, we thought first year high school students would be the most appropriate subject pool for evaluating early functional changes related to PeVA. Students who reported current experience of PeVA were excluded to focus on the effect of past PeVA experiences on brain function. Other exclusion criteria for all subjects were previous/current psychiatric history or sexual/physical abuse history, full-scale IQ less than 70, significant head trauma or brain disease history, or any magnetic resonance imaging contraindication.

\section{Behavioral measurements}

Exposure to parent or peer verbal abuse during elementary and middle school periods was assessed using the Verbal Abuse Questionnaire (VAQ), which evaluates 15 kinds of verbal abuse. A VAQ score of 40 or higher represents substantial exposure to PeVA. ${ }^{3}$ This cut-off score of 40 was acceptable in our recent study with 5814 Korean university students. ${ }^{28}$ Additionally, to verify the validity of the cutoff value, we used cluster analysis using the scores of PeVA and PaVA, and this cutoff score of 40 was generally acceptable to our data (Supplementary Text 1, Figure 1 in the online-only Data Supplement). Therefore, we adopted a cutoff value of 40 to define the high-PeVA group. Subjects who were scanned by fMRI were divided into two groups consisting of 11 high-PeVA and 12 low-PeVA subjects. Anxiety and depression were evaluated using the State-Trait Anxiety Inventory (STAI) ${ }^{29}$ and Beck Depression Inventory (BDI). ${ }^{30}$ The usage frequency of swear words in peer relationships was measured using a 5 -point Likert scale $(0-4,0=$ none, $1=$ once or twice a month, $2=$ once or twice a week, $3=$ once or twice a day, $4=$ almost always). A Mann-Whitney $U$ test was used to measure the differences in behavioral features between the high-PeVA and low-PeVA groups.

\section{Procedures}

We recruited 32 first year healthy male students from several 
high schools. All subjects were asked to complete a survey that contained questions related to behavior measurements using web-based questionnaires. Thirty participants completed the survey and 25 of them finally agreed to have fMRI scanning.

All 25 subjects accomplish our experiments along identical process. The experimenters explain fMRI task paradigms, and subjects conducted fMRI scanning. Also, two psychologists interviewed with participants to get diagnostic information of psychiatric disorders using the Kiddie-Schedule for Affective Disorders and Schizophrenia-Present and Lifetime VersionKorean Version (K-SADS-PL-K). After psychologists interviewed the participants, three authors in our study (one child psychiatrist and two psychiatrists) shared the information of participants to exclude participants who have psychiatric disorder. The Wechsler Intelligence Scale for Children-Revised (WISC-R) was used on participants to evaluate the intelligence of the subjects in the study. Among the 25 subjects, one subject with teeth braces was excluded and the data of one subject who exhibited much movement during fMRI due to rhinitis was excluded in the analysis. Finally, we obtained fMRI results from 23 subjects. All subjects joined voluntarily, and we received written informed consent from both the subjects and their parents for each step of study (behavioral measurements and fMRI). The study was approved by the Ethics Committee of the Korea Advanced Institute of Science and Technology (KAIST).

\section{fMRI experimental design}

A modified Emotional Stroop task was designed to measure the implicit processing-related brain activity to emotional words. The task consisted of four different conditions of word stimuli: swearing (SWE), negative (NEG), positive (POS) and neutral (NEU). To choose appropriate word stimuli, sixty words per each condition were screened. Swear words were selected from a survey on the use of language in adolescence by the National Institute of the Korean Language in 2011. ${ }^{6}$ Other emotional words were chosen from several Korean articles related to emotional language. ${ }^{31-34}$ Using a questionnaire of 240 words, emotional valence and type were evaluated by thirty high school students who did not participate in the fMRI study. Emotional valence was evaluated by a 10-point Likert scale (0-9, 9 represents the highest emotional valence) and the type of emotional word was selected as one from six different choices (positive, anger, fear, disgust, sad, and neutral). Negative words were selected when four out of five students reported fear, disgust, or anger feelings because we believed swearing words may induce these kinds of emotions. Finally, 12 words per condition were selected. The mean valence of swear words (5.62) was similar to that of negative words [5.59, t (22) $=0.190, \mathrm{p}=0.85]$; there were no significant differences between negative and positive words $[5.27, \mathrm{t}(22)=$
1.688, $\mathrm{p}=0.11]$ in emotional valence. Neutral words had a small mean emotional valence $(0.83)$. There were no differences between conditions according to the number of letters and number of syllables.

During the fMRI session, the subjects performed the modified Emotional Stroop task. The four different conditioned words (SWE, NEG, POS and NEU) with three different colors (red, blue, and yellow) were represented for $0.75 \mathrm{~s}$ followed by a black screen for $3.25 \mathrm{~s}$ in a pseudorandomized order; subjects were asked to press buttons of an MRI-compatible response box with the right index, middle and ring finger according to color of the words. In addition, 45 color words with the same color (COLOR) were inserted to maintain attention during the intervals of the target stimuli.

Reaction time and error rate date were analyzed using 2 by 4 repeated measures one-way analysis of variance (RANOVA) to measure the effects of group, condition and group by trial. Incorrect responses and individual delayed response time ( $>2 \mathrm{SD}$ from a subject's mean) were excluded for reaction time analysis. ${ }^{17,35}$

\section{Image acquisition}

A total 200 blood oxygen level dependent based functional MRI images were acquired at 3.0 Tesla (Siemens) with 12 channel head coil using $\mathrm{T} 2{ }^{*}$-weighted gradient echo, echo-planar imaging pulse sequence ( 35 axial slices, $\mathrm{TR}=2200 \mathrm{~ms}, \mathrm{TE}=30$ $\mathrm{ms}$, flip angle $=90$ degrees, $\mathrm{FOV}=192 \times 192 \mathrm{~mm}$, acquisition matrix $=64 \times 64$, slice thickness $=3 \mathrm{~mm}$, voxel size $=3 \times 3 \times 3.3$ $\mathrm{mm})$. A high resolution structural image was obtained with a T1 weighed sequence $(\mathrm{TI}=900 \mathrm{~ms}, \mathrm{TR}=1800 \mathrm{~ms}, \mathrm{TE}=2.52 \mathrm{~ms}$, Flip angle $=9$ degrees, FOV $=256 \times 256 \mathrm{~mm}$, slice thickness $=1$ $\mathrm{mm}$, voxel size $=1 \times 1 \times 1 \mathrm{~mm}$ ) before fMRI scanning.

\section{fMRI analysis}

All images were preprocessed using FMRI Expert Analysis Tool (FEAT) in the FMRIB Software Library (FSL). ${ }^{36}$ EPI images were temporally high-pass filtered with a cutoff frequency $0.01 \mathrm{~Hz}$ to remove low-frequency drift and spatially smoothed using an $8 \mathrm{~mm}$ full-width/half-maximum Gaussian Kernel. Motion correction was performed using the linear interpolation method (MCFLIRT). Skull and non-brain areas of both anatomical and functional images were removed using a brain extraction tool (BET), and threshold values of anatomical images were searched manually to get satisfactory results. Registration from functional EPI images to T1 weighted anatomical volumes was conducted with 7 degrees of freedom, and T1 weighted anatomical images were registered to $2 \mathrm{~mm}$ MNI standard space with 12 degrees of freedom using FMRIB's Linear Image Registration Tool (FLIRT).

After preprocessing, single and group level fMRI analyses 
were performed with FEAT in the FMRIB Software Library (FSL). ${ }^{36}$ SWE, NEG, POS, NEU, and COLOR variables with their temporal derivatives were modeled; all variables were convolved with a double gamma hemodynamic response function (HRF). A general linear model (GLM) was applied to measure parameter estimates of each condition. Also, motion parameters generated from motion correction step in preprocessing were added into GLM as confound variables. To assess differences in brain activity among SWE, NEG, POS and NEU conditions, the RANOVA applying the mixed effects model was conducted. Gaussian random field theory was applied to correct for multiple comparisons at the cluster-level. Threshold for statistical significance is a family-wise error corrected cluster threshold $\mathrm{p}<0.05$. From the RANOVA results, we extracted mean of parameter estimates using $10 \mathrm{~mm}$ diameter sphere around peak coordinate of each brain region to measure trends of activation patterns in these regions according to different kinds of emotional stimuli and to find the region more activated in SWE than in other emotional stimuli.

Additionally, an unpaired t-test applying the mixed effects model was performed using the parameter estimates of three emotional contrasts (SWE $>\mathrm{NEU}, \mathrm{NEG}>\mathrm{NEU}$, and $\mathrm{POS}>\mathrm{NEU}$ ) of each subject to assess differences between high-PeVA and low-PeVA group, and frequency of swear words exposure was included as covariate in this analysis.

\section{Functional connectivity}

In the study, we used a beta series correlation method ${ }^{37}$ to measure the PeVA effects on swearing-task specific FLN connections between the left VLPFC and left amygdala and left hippocampus. Using FEAT in FSL, ${ }^{36}$ the beta value of every trial event of four conditions was calculated, thus, 12 beta series $\times 4$ conditions (SWE, NEG, POS, and NEU) were acquired in each ROI. Six motion parameters generated from MCFLIRT in the preprocessing step were added as confound variables to reduce the effects of head movement on beta series since head movement can affect the results of functional connectivity. ${ }^{38,39}$ In addition, the mean displacements of head position were measured to find differences of the degrees of head movements between the high-PeVA group and the lowPeVA group; there were no significant differences in head movement between the groups (Supplementary Table 1 in the online-only Data Supplement). After getting the beta map of every trial event, each beta map was registered to an

Table 1. The results of Mann-Whitney $U$ test of behavioral measurements between high-PeVA and low-PeVA group in MRI participants

\begin{tabular}{|c|c|c|c|c|c|c|}
\hline \multirow{3}{*}{ Variables } & \multicolumn{4}{|c|}{ Participants } & \multirow{2}{*}{\multicolumn{2}{|c|}{ Analysis }} \\
\hline & \multicolumn{2}{|c|}{ High-PeVA $(\mathrm{N}=11)$} & \multicolumn{2}{|c|}{ Low-PeVA $(\mathrm{N}=12)$} & & \\
\hline & Mean & SD & Mean & $\mathrm{SD}$ & $\mathrm{Z}$ & $\mathrm{p}$ \\
\hline Age & 16.24 & 0.49 & 16.13 & 0.42 & -0.431 & 0.695 \\
\hline Frequency of swearing* & 4.64 & 3.01 & 3.58 & 2.64 & -0.933 & 0.379 \\
\hline Parental VAQ & 41.27 & 20.71 & 23.33 & 8.91 & -2.995 & 0.002 \\
\hline Peer VAQ & 59.91 & 14.27 & 24.58 & 8.06 & -4.066 & $<0.001$ \\
\hline STAI-state & 41.82 & 5.60 & 38.33 & 10.13 & -0.894 & 0.379 \\
\hline STAI-trait & 47.45 & 5.94 & 44.25 & 7.75 & -1.392 & 0.169 \\
\hline $\mathrm{BDI}$ & 7.91 & 4.30 & 3.50 & 3.55 & -2.535 & 0.011 \\
\hline Total IQ & 110.00 & 8.72 & 109.83 & 15.56 & -0.339 & 0.740 \\
\hline Verbal IQ & 112.36 & 11.31 & 110.42 & 12.41 & -0.154 & 0.880 \\
\hline Performance IQ & 104.36 & 8.99 & 99.25 & 31.87 & -0.092 & 0.928 \\
\hline \multicolumn{7}{|l|}{ Stroop task $\left(\mathrm{N}=22^{\dagger}\right)$} \\
\hline SWE RT (s) & 0.59 & 0.06 & 0.62 & 0.17 & -0.396 & 0.722 \\
\hline SWE ER (\%) & 1.67 & 3.51 & 4.77 & 9.27 & -0.539 & 0.722 \\
\hline NEG RT (s) & 0.63 & 0.07 & 0.62 & 0.14 & -0.528 & 0.628 \\
\hline NEG ER (\%) & 6.82 & 8.81 & 4.57 & 6.17 & -0.363 & 0.771 \\
\hline POS RT (s) & 0.62 & 0.07 & 0.61 & 0.15 & -1.055 & 0.314 \\
\hline POS ER (\%) & 0.00 & 0.00 & 4.44 & 6.33 & -2.257 & 0.107 \\
\hline NEU RT (s) & 0.59 & 0.06 & 0.59 & 0.14 & -0.989 & 0.323 \\
\hline NEU ER (\%) & 0.00 & 0.00 & 5.90 & 7.16 & -2.525 & 0.050 \\
\hline
\end{tabular}

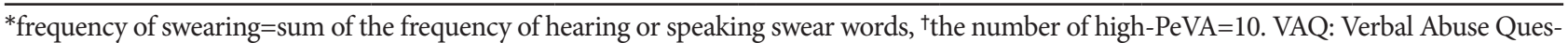
tionnaire, STAI: Stait-Trait Anxiety Inventory, BDI: Beck Depression Inventory, RT: reaction time, ER: error rate, PeVA: peer verbal abuse group, MRI: magnetic resonance imaging, IQ: intelligence quotient, SWE: swearing, NEG: negative, POS: positive, NEU: neutral words 
MNI standard space. The functional ROIs represented the left VLPFC, and the left amygdala and left hippocampus were selected from the activation maps of SWE vs. NEU in the RANOVA results. Each ROI's beta values were measured using a $10 \mathrm{~mm}$ in diameter mask around peak coordinates described in Supplementary Table 2 (in the online-only Data Supplement) (MNI coordinates (X/Y/Z) of left VLPFC $=-42 /$ $24 /-8$, left amygdala $=-20 /-8 /-14$ and left hippocampus $=-20 /$ $-30 /-8)$. To reduce the effects of outliers, non-parameteric method was applied. Using the Spearman correlation between the beta series of two ROIs in SWE, NEG, POS and NEU contrast, the functional interactions of the left VLPFC-left amygdala, left VLPFC-left hippocampus, and left amygdala-left hippocampus were quantified at the individual level. The effects of group, trial and group by trial interaction on the connectivity were verified using RANOVA. In addition, to evaluate the effects of changes in brain functional connectivity on subjects' behavior, the relationship between psychiatric symptom scores and FLN connectivity was measured using the Spearman correlation.

\section{RESULTS}

\section{Behavioral measurements}

The results of the Mann-Whitney $\mathrm{U}$ test of the 23 subjects who participated in fMRI scanning are shown in Table 1. Among the various measurements, the ratings of PaVA ( $p=$
$0.002)$ and depressive symptoms $(\mathrm{p}=0.011)$ were greater in the high-PeVA group than in the low-PeVA group. There were no differences in the swearing usage frequency, IQ and age between the two groups. The results of the total of 30 subjects are represented in Supplementary Table 3 (in the online-only Data Supplement).

In addition, reaction time and error rate during emotional Stroop task were evaluated using RANOVA. The data of 22 subjects (10 high-PeVA and 12 low-PeVA) were included and one high-PeVA data was missed by the error of button box. The trial effect on reaction time was significant $[F(3,60)=$ $5.518, \mathrm{p}=0.002$ ]. In post hoc paired t-test, all emotional stimuli showed a delayed response time compared to NEU (all ps<0.05) (Supplementary Figure 2 in the online-only Data Supplement), and there was no difference between emotional stimuli. Both group effect and group by trial interaction did not have a significant effect $[\mathrm{F}(1,20)=0.03, \mathrm{p}=0.96$ and $\mathrm{F}(3$, $60)=1.345, p=0.27$, respectively]. In error rate analysis, there was no significant effect of group $[\mathrm{F}(1,20)=2.764, \mathrm{p}=0.11]$, trials $[\mathrm{F}(3,60)=1.534, \mathrm{p}=0.22]$ and group by trials $[\mathrm{F}(3$, $60)=2.130, p=0.11]$. Mean values of reaction time and error rate were described in Table 1.

\section{The overall brain activity patterns induced by emotional word stimuli}

The activity of several brain regions that are related to emotion processing showed hyper-activation during SWE com-

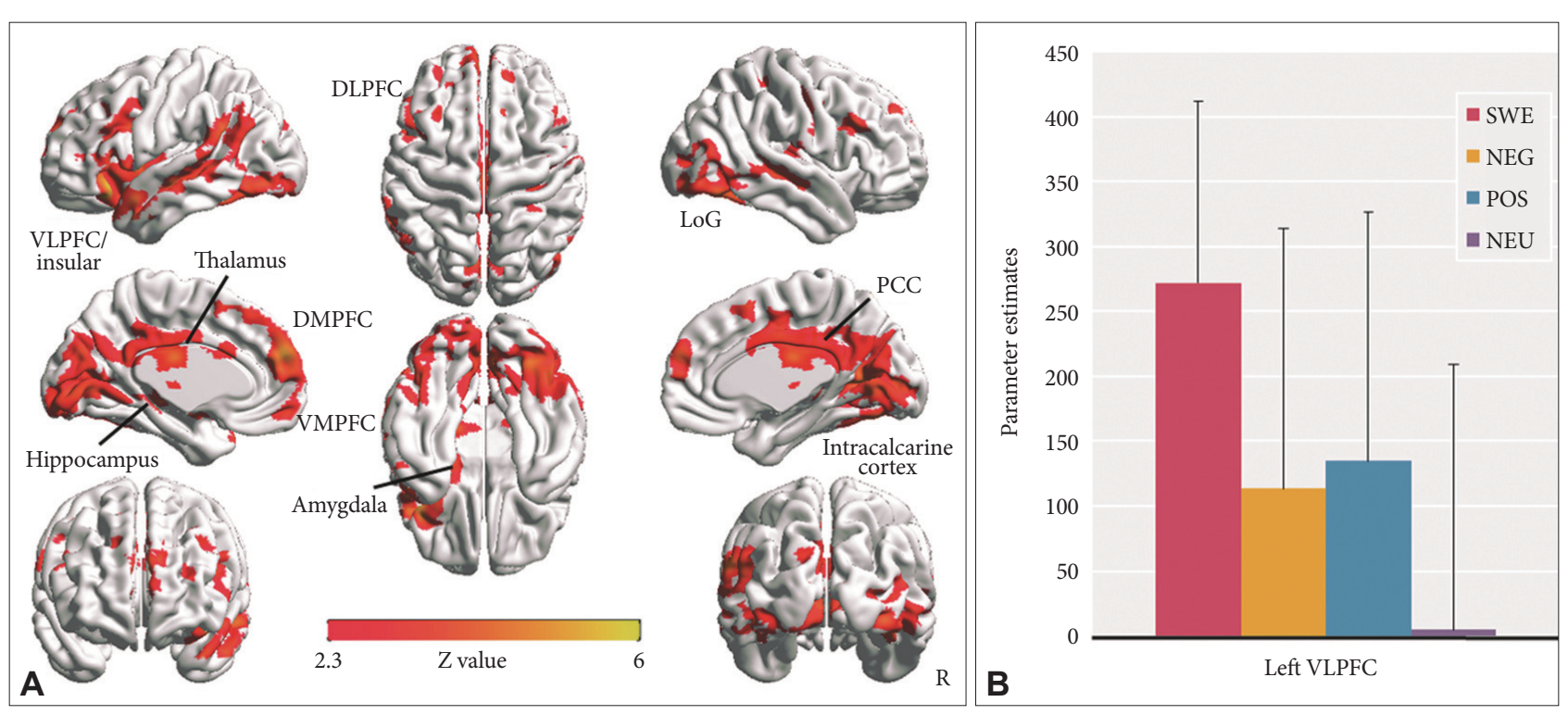

Figure 1. Brain regions showed more activity during SWE than NEU in repeated ANOVA. A: Several brain regions including left VLPFC, left insular and left amygdala were more activated during SWE compared to NEU $(Z>2.3$, corrected $p<0.05)$. B: Among various brain regions, the left VLPFC activities were greater in SWE than other emotional stimuli $[F(3,66)=14.49, p<0.001]$. The parameter estimates of the left VLPFC were extracted using a $10 \mathrm{~mm}$ sphere around the peak MNI coordinates (X/Y/Z) (-42/24/-8). R: right, VLPFC: ventrolateral prefrontal cortex, DLPFC: dorsolateral prefrontal cortex, DMPFC: dorsomedial prefrontal cortex, VMPFC: ventromedial prefrontal cortex, LOC: lateral occipital cortex, PCC: posterior cingulate cortex, SWE: swearing, NEU: neutral words, ROI: region of interest, MNI: Montreal Neurological Institute. 
pared to NEU $(\mathrm{Z}>2.3$, corrected $\mathrm{p}<0.05)$ (Figure $1 \mathrm{~A})$. More detailed activated brain regions and activation patterns of these regions using extracted parameter estimates are shown in Supplementary Figure 3, Table 3 (in the online-only Data Supplement). The activation of these regions presented a linear trend among SWE, NEG/POS and NEU. Furthermore, left VLPFC activity showed significant differences among emotional words stimuli $[\mathrm{F}(3,66)=14.49, \mathrm{p}<0.001]$; in post hoc analysis, hyperactivity in SWE compared to other emotional stimuli had these differences: $95 \% \mathrm{CI}=70.42$ to $247.27, \mathrm{p}<0.001$ in SWE $>$ NEG and $95 \% \mathrm{CI}=46.84$ to $228.15, \mathrm{p}<0.001$ in $\mathrm{SWE}>$ POS contrasts (Figure 1B). There was no region that shows significant activity in NEG $>$ SWE and POS $>$ SWE contrasts.
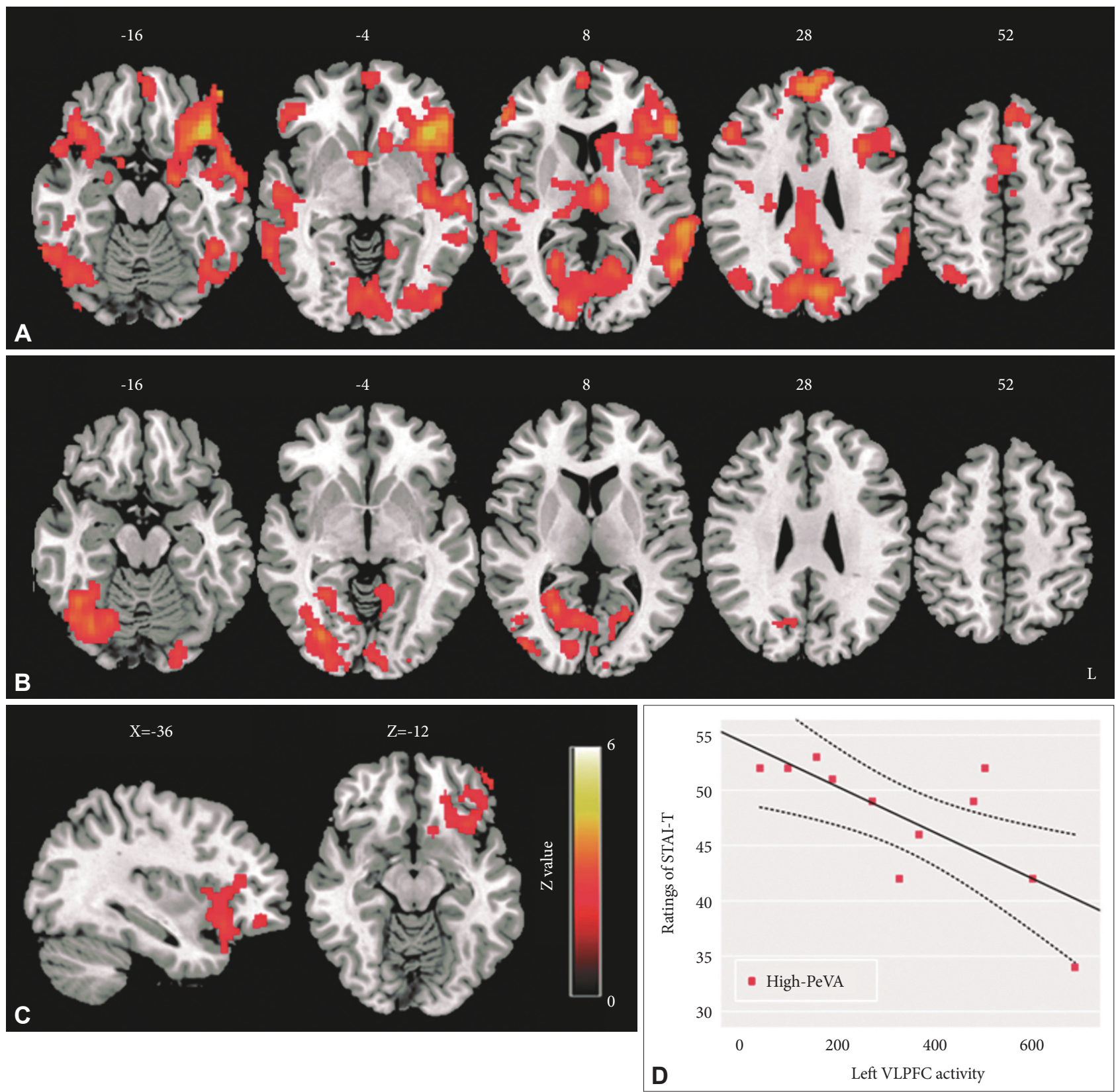

Figure 2. Regions showed hyperactivity in the high-PeVA group compared to the low-PeVA group in SWE>NEU. A: In the SWE vs. NEU contrast, the high-PeVA group showed activity of several brain regions including the left insular, VLPFC and visual information processing areas, $(B)$ and the low-PeVA group represented visual cortex activity $(Z>2.3$, corrected $p<0.05)$. C: Compared with the low-PeVA group, the high-PeVA group exhibited greater activity in the left insular and left ventrolateral prefrontal cortex (VLPFC) after controlling for the frequency of usage of swear words $(Z>2$, corrected $p<0.05$ ). D: Additionally, the activity of the left VLPFC extracted by a $10 \mathrm{~mm} \mathrm{ROI} \mathrm{(peak} \mathrm{MNI} \mathrm{co-}$ ordinates $=-42 / 24 /-8)$ was negatively correlated with ratings of an anxiety trait $(p=0.015) .95 \%$ of confidence interval is depicted by a dotted line. PeVA: peer verbal abuse, STAI-T: Stait-Trait Anxiety Inventory, SWE: swearing, NEU: neutral words, ROI: region of interest, MNI: Montreal Neurological Institute. 


\section{The differences in brain activity between high-PeVA and low-PeVA group during swear word processing}

In the high-PeVA group, SWE stimuli induced significant activation in several regions including the bilateral VLPFC, left insular, bilateral amygdala, bilateral dorsolateral prefrontal cortex (DLPFC) and intracalcarine cortex (SWE $>$ NEU contrast, $\mathrm{Z}>2.3$, corrected $\mathrm{p}<0.05$ ) (Figure $2 \mathrm{~A}$ ). The high-PeVA group had higher activation in the left insular and left VLPFC compared to the low-PeVA group (Figure 2B) in SWE $>$ NEU contrast after controlling for the frequency of usage of swear words (Figure 2C). In NEG $>$ NEU and POS $>$ NEU contrasts, there were not any significant differences in activation maps between the high-PeVA and low-PeVA groups $(\mathrm{Z}>2$, corrected $\mathrm{p}<0.05)$.

To find the significance of the left VLPFC hyper-activation during swear word processing, the correlations between various behavioral measurements and the left VLPFC activity were evaluated. In the high-PeVA group, the activity of the left VLPFC was negatively associated with an anxiety trait (STAIT) (spearman's rho $=-0.705, \mathrm{p}=0.015$ ) (Figure 2D), but this correlation was not shown in the low-PeVA group (spearman's $\mathrm{rho}=0.291, \mathrm{p}=0.358$ ).

\section{Differences in functional connectivity among the left VLPFC, left amygdala, and left hippocampus between the high-PeVA group and low-PeVA group}

\section{Left VLPFC-left hippocampus connectivity}

We assessed group differences in the left VLPFC-left hippocampus using RANOVA. The group effect on the left VLP-
FC-left hippocampus was significant $[\mathrm{F}(1,21)=7.777, \mathrm{p}=$ $0.011]$, and the effects of trials and group by trial interaction did not have a significant effect on left VLPFC-left hippocampus connectivity $[\mathrm{F}(3,63)=1.565, \mathrm{p}=0.21$ and $\mathrm{F}(3,63)=$ $1.032, \mathrm{p}=0.39$, respectively]. In post-hoc analysis using a t-test with Bonferroni correction for multiple comparisons (corrected $\mathrm{p}=0.05 / 4$ or 0.0125 ), the high-PeVA group had a significantly higher left VLPFC-left hippocampus connectivity than the low-PeVA group in SWE conditions [ $t(21)=2.759, \mathrm{p}=$ 0.012 ] and a trend level difference in connectivity in NEG compared to the low-PeVA group [ $\mathrm{t}(21)=2.323, \mathrm{p}=0.03$ ] (Figure $3 \mathrm{~A})$.

\section{Left VLPFC-left amygdala connectivity}

The RANOVA analysis revealed a significant effect of group $[\mathrm{F}(1,21)=4.801, \mathrm{p}=0.04]$ on left VLPFC-left amygdala connectivity. There was no significant effect of trials [F (3, $63)=1.520, \mathrm{p}=0.22]$ and group by trials interaction $[\mathrm{F}(3$, $63)=0.875, p=0.46]$. In post-hoc analysis, there was no significant group difference in functional connectivity between the left VLPFC and left amygdala among SWE, NEG, POS and NEU stimuli. There was only a trend of higher connectivity in the high-PeVA group than in the low-PeVA group in NEG and NEU contrast $[\mathrm{t}(21)=2.054, \mathrm{p}=0.053$ and $\mathrm{t}(21)=2.099$, $\mathrm{p}=0.048$, respectively] (Supplementary Figure 4 in the onlineonly Data Supplement).

\section{Left amygdala-left hippocampus connectivity}

There was no significant effect of group $[\mathrm{F}(1,21)=2.916$, $\mathrm{p}=0.1]$, trials $[\mathrm{F}(3,63)=0.741, \mathrm{p}=0.53]$ and group by trials $[\mathrm{F}$
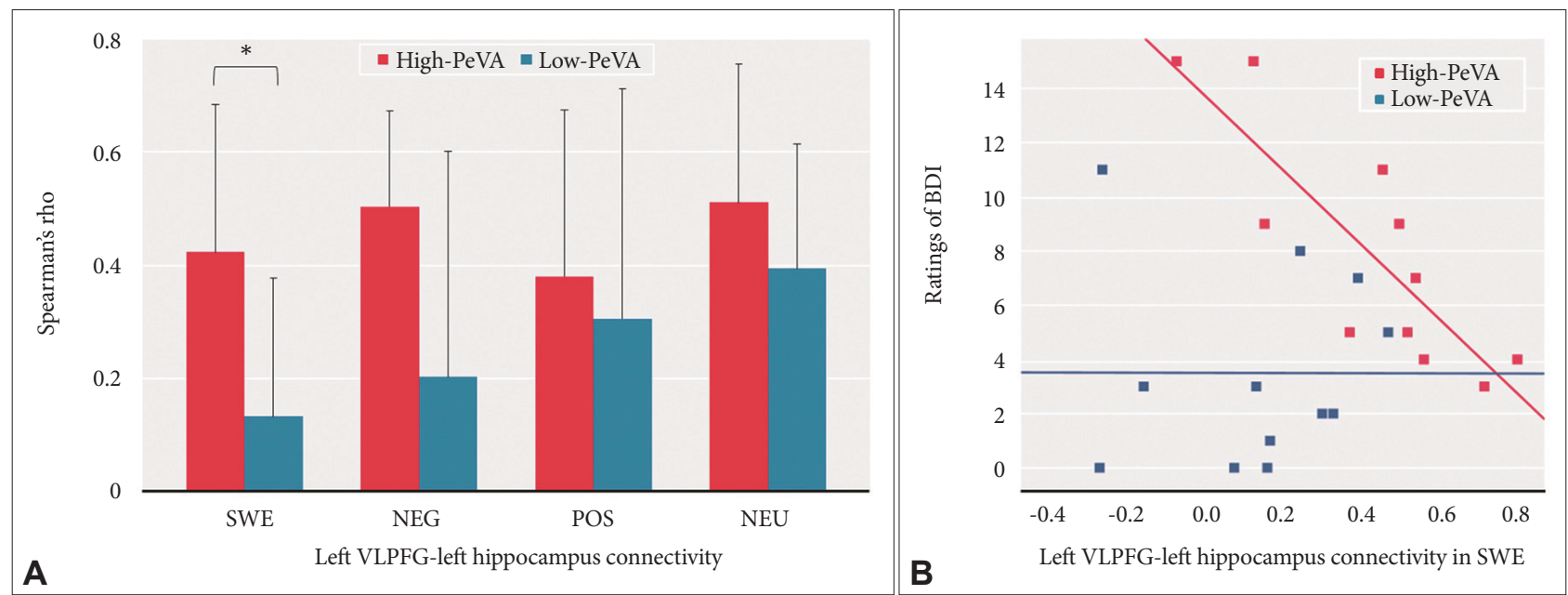

Figure 3. The functional connectivity patterns between the left VLPFC and the left hippocampus. A: The overall functional connectivity of the left VLPFC-left hippocampus during the modified emotional Stroop task were greater in the high-PeVA group than in the low-PeVA group $(F=7.777, p=0.011)$. The high-PeVA group had significantly higher left VLPFC-left hippocampus connectivity during SWE stimuli compared to the low-PeVA group ( ${ }^{*} \mathrm{p}=0.012$ ) and a trend of higher connectivity in NEG stimuli than the low-PeVA group ( $p=0.03$ ). B: Moreover, in the high-PeVA group, the ratings of BDI were negatively associated with the connectivity of the left VLPFC-left hippocampus in SWE $(p=0.001)$. PeVA: peer verbal abuse, BDI: Beck depression inventory, VLPFC: ventrolateral prefrontal cortex, SWE: swearing, NEG: negative words. 
$(3,63)=0.539, \mathrm{p}=0.66]$ on left amygdala-left hippocampus connectivity (Supplementary Figure 4 in the online-only Data Supplement).

\section{The relationship between the left VLPFC-left hippocampus connectivity and ratings of depression}

To find the possible relationship between the changes of functional connectivity and behavioral measurements, we evaluated the correlations between the left VLPFC-left hippocampus connectivity and behavioral measures. Within several behavioral measures, the ratings of BDI were highly correlated with the left VLPFC-left hippocampus connectivity in the high-PeVA group both at SWE and NEG conditions (Spearman's rho $=-0.867, \mathrm{p}=0.001$ and Spearman's $r h o=-0.610, \mathrm{p}=$ 0.046 , respectively). However, in the low-PeVA group, the left VLPFC-left hippocampus connectivity during negative emotional word processing did not show any significant correlation with BDI (Figure 3B) (Supplementary Figure 5 in the online-only Data Supplement).

\section{DISCUSSION}

In our study, although subjects had no overt psychiatric symptoms, adolescents in the high-PeVA group presented more depressive symptom scores, and had a higher frequency of previous PaVA experiences compared to low-PeVA group. Moreover, the high-PeVA group had greater activity of the VLPFC, negatively related to the anxiety trait, and higher functional interaction between the left VLPFC and left hippocampus, negatively related to the severity of depressive symptoms, during implicit processing to swear word. Our preliminary results, showing the exposure to PeVA links to functional changes in the left frontolimbic network which are related to psychiatric symptoms, suggest that swear words from peers can harm children even if the hurt was unintended or not overtly used.

In behavioral results, although frequency of exposure to swear words showed no group difference, PeVA experiences were closely related with PaVA experiences. A previous study reported that parental maltreatment can be a risk factor for victimization by peers. ${ }^{40}$ It is still unclear how the two verbal abuses, PaVA and PeVA, are closely related. However, it is possible that children who were verbally mistreated by their parents did not have positive self-esteem, and they could not establish healthy defense mechanisms to keep themselves away from peer bullying. ${ }^{41}$ Also, in emotional Stroop task, although interference effect of emotional stimuli were reported similar with previous study, ${ }^{35}$ there was no group effect on reaction time and error rate. None of our subject reported a significant psychiatric problem in constructed interview (K-SADS-PL-K), and it can affect our behavioral results. In previous study using emotional Stroop task, preclinical subjects did not show significant difference with normal control. ${ }^{42}$

In the fMRI analysis, the brain regions engaged in emotion processing, ${ }^{43}$ including the VLPFC, DLPFC, amygdala and hippocampus, showed linear trend in activation (SWE $>\mathrm{NEG}>$ $\mathrm{NEU}$ ) although there were no significant differences between swear words and negative words in emotional valence $(\mathrm{p}=$ 0.85 ). Thus, the swear words have more impact on adolescents' brain than what they think.

Within various brain regions that were activated in SWE> NEU, the VLPFC was significantly more activated in highPeVA group than low-PeVA group. In recent meta-analysis, the left inferior frontal gyrus including the VLPFC was activated by basic negative emotional stimuli; disgust and anger stimuli induced a higher level of activation in this region compared to sadness. ${ }^{44}$ While the VLPFC was involved in basic emotion processing, group differences were observed only in SWE $>$ NEU, not NEG $>$ NEU contrasts. The characteristics of the VLPFC and our preclinical subjects can affect these results. In patients with major depressive disorder, hyperactivity in the VLPFC during automatic emotional regulation is frequently reported. ${ }^{45}$ Furthermore, previous studies reported that the VLPFC can be closely linked with the process of emotion regulation associated with individual's experiences. Role of the VLPFC during emotion regulation is associated with voluntary processes, ${ }^{43,45}$ such as emotion reappraisal ${ }^{15,16,46}$ and suppression. ${ }^{47,48}$ In addition, retrieval of autobiographic memory, ${ }^{20,49}$ or processing trauma-related information ${ }^{19}$ can induce the VLPFC activity. Therefore, role of the VLPFC in high-PeVA group seems to associate with control mechanism to reduce the negative emotional effects triggered by swear words that were closely connected to previous PeVA experiences. Our negative correlation result between the VLPFC activity and anxiety trait can support this explanation, and previous studies also reported top-down regulatory process of the VLPFC that is related to anti-anxiety effects. ${ }^{13,50,51}$

In previous fMRI studies, increased amygdala-responsiveness to negative stimuli was commonly reported in the victims of childhood maltreatments. ${ }^{52-57}$ In our study, although we did not find any significant difference in amygdala-responsiveness between high-PeVA and low-PeVA groups, bilateral amygdala were activated during swearing stimuli in high-PeVA group. Our subjects were preclinical, and no subject reported significant psychiatric symptoms, even in high-PeVA group. Therefore, subjects who had prominent problems in regulatory mechanism on the amygdala activity might be excluded. Additionally, types of stimuli can be related with our results. While all of the previous studies mentioned above used face stimuli as the negative emotional stimuli, we selected emo- 
tional words selected using a preliminary survey by high school students. One recent study with complex PTSD using words stimuli also did not report the amygdala hyperactivity. ${ }^{58}$

During swear word processing, functional connection between the left hippocampus and the left VLPFC was more enhanced in the high-PeVA group than in the low-PeVA group. The hippocampal region and the lateral prefrontal cortex are anatomically connected ${ }^{59}$ however, the specific function of this network is yet unclear. Our functional ROI of the left hippocampus was located in the posterior part of the hippocampus; in comparative studies, the posterior hippocampal region also has direct afferent and indirect efferent connections with the lateral prefrontal cortex. ${ }^{60,61}$ Previous studies reported that the posterior part of the hippocampus seems to be more involved in memory retrieval than in encoding; ${ }^{62-64}$ the function of the left side of the hippocampus is related to meaningful verbal memory encoding and retrieval. ${ }^{65,66}$ Thus, the left posterior hippocampus region may be closely related to memory retrieval associated with PeVA experiences, and the VLPFC may guide the selection of relevant memory retrieval through a top-down process. ${ }^{67}$ The VLPFC-hippocampus connection is related to controlled retrieval. ${ }^{68}$ The children who have traumatic memories related to swear words can be easily re-traumatized by being exposed to swear words and associated memories; thus they might use more energy to control their unhappy memory through the left VLPFC-left hippocampus network. Additionally, in the high-PeVA group, the activity of this network played a role in reducing their depressive symptoms. Therefore, this network may be a compensatory mechanism to manage their traumatic experience. However, the role of the left VLPFC-left hippocampus on depressive symptoms should be clarified in future study because subjects in our preclinical study represented low BDI scores even in high-PeVA group (mean=7.91).

There are some limitations that should be noted. First, our sample size was rather small for evaluating correlations between psychiatric symptoms and activity or connectivity of the brain regions. However, we recruited adolescents who had recently graduated middle school, a developmentally sensitive period, ${ }^{3}$ but were easily exposed to swear words. Also, to exclude the effects of psychiatric symptoms on the behavior and the brain network of the adolescents, only those subjects who had no significant psychiatric symptoms were included in the study. Therefore, our study can give preliminary evidence to understand the functional brain mechanism in the victims of PeVA. Second, although we set the cutoff score based on a previous study, ${ }^{3,28}$ the cutoff score of 40 was still yet somewhat arbitrary. However, we hypothesized and tried to show that the role of a specific brain region or a network can be different in high-PeVA and low-PeVA subjects. It was difficult to divide subjects into two distinct groups, since PeVA is not a psychiatric disease that can be diagnosed. Thus, to compensate this limitation, we verified the cut-off score using a cluster analysis, and found that the cut-off score was acceptable in our previous study with 5814 young adults. ${ }^{28}$ Nonetheless, this cutoff score can be confined to young adults, such as university students, and it can cause bias on our results. Third, PeVA was assessed using self-administrated questionnaire that may have a retrospective memory problem. We believe PeVA may be a type of painful memory that is reflected from what adolescents felt rather than what they experienced. Our results may be more confirmative if objective measurements, such as evaluation of subjects' parents or teacher, were included in this study. An increased activity of the FLN, shown in our results, needs to be confirmed by further longitudinal studies with subjects with a wider age range. Sex and age effects on brain function changes in the PeVA group should be clarified in a further study.

In spite of these limitations, our preliminary results indicated that the VLPFC and left VLPFC-left hippocampus network were important in processing swear words in high-PeVA adolescents. The victims of PeVA seems to require more resources to control the stimuli that were related to previous negative experiences, even if it is implicitly presented, and the disruption of the regulatory processes may lead anxiety and depressive symptoms. We think these results may be able to provide the clues in understanding how verbal abuse experiences can induce depressive symptoms and be the possible answer to the question: "Why should adolescents not use swear words without any concern for their peers?"

\section{Supplementary Materials}

The online-only Data Supplement is available with this article at https://doi.org/10.4306/pi.2017.14.4.441.

\section{Acknowledgments}

This work was supported by grants (NRF-2006-2005372 to B. Jeong) from National Research Foundation, BK21 plus, the KAIST Future Systems Healthcare Project, and KI for Health Science and Technology Project Grant [N11160068, N11160074], and by in part grant from Brain research institute. The authors thank Y.-B. Lee and S.Cho for their help to our fMRI experiment.

\section{REFERENCES}

1. Hart H, Rubia K. Neuroimaging of child abuse: a critical review. Front Hum Neurosci 2012;6:52.

2. Teicher MH, Samson JA, Polcari A, McGreenery CE. Sticks, stones, and hurtful words: relative effects of various forms of childhood maltreatment. Am J Psychiatry 2006;163:993-1000.

3. Teicher MH, Samson JA, Sheu YS, Polcari A, McGreenery CE. Hurtful words: association of exposure to peer verbal abuse with elevated psychiatric symptom scores and corpus callosum abnormalities. Am J Psychiatry 2010;167:1464-1471.

4. Choi J, Jeong B, Polcari A, Rohan ML, Teicher MH. Reduced fractional 
anisotropy in the visual limbic pathway of young adults witnessing domestic violence in childhood. Neuroimage 2012;59:1071-1079.

5. Andersen SL, Tomada A, Vincow ES, Valente E, Polcari A, Teicher MH. Preliminary evidence for sensitive periods in the effect of childhood sexual abuse on regional brain development. J Neuropsychiatry Clin Neurosci 2008;20:292-301.

6. Chang KH. A National Survey on Language Use and Language Attitude in Adolescents. Seoul: The National Institute of The Korean Language; 2011

7. Wang J, Iannotti RJ, Nansel TR. School bullying among adolescents in the United States: physical, verbal, relational, and cyber. J Adolesc Health 2009;45:368-375.

8. Nansel TR, Overpeck M, Pilla RS, Ruan WJ, Simons-Morton B, Scheidt P. Bullying behaviors among US youth - Prevalence and association with psychosocial adjustment. JAMA 2001;285:2094-2100.

9. Maughan A, Cicchetti D. Impact of child maltreatment and interadult violence on children's emotion regulation abilities and socioemotional adjustment. Child Dev 2002;73:1525-1542.

10. Schelble JL, Franks BA, Miller MD. Emotion Dysregulation and Academic Resilience in Maltreated Children. Child Youth Care Forum 2010;39:289-303.

11. Eluvathingal TJ, Chugani HT, Behen ME, Juhasz C, Muzik O, Maqbool M, et al. Abnormal brain connectivity in children after early severe socioemotional deprivation: a diffusion tensor imaging study. Pediatrics 2006;117:2093-2100.

12. Choi J, Jeong B, Rohan ML, Polcari AM, Teicher MH. Preliminary evidence for white matter tract abnormalities in young adults exposed to parental verbal abuse. Biol Psychiatry 2009;65:227-234.

13. Burghy CA, Stodola DE, Ruttle PL, Molloy EK, Armstrong JM, Oler JA, et al. Developmental pathways to amygdala-prefrontal function and internalizing symptoms in adolescence. Nat Neurosci 2012;15:1736-1741.

14. Morandotti N, Dima D, Jogia J, Frangou S, Sala M, De Vidovich GZ, et al. Childhood abuse is associated with structural impairment in the ventrolateral prefrontal cortex and aggressiveness in patients with borderline personality disorder. Psychiatry Res 2013;213:18-23.

15. Buhle JT, Silvers JA, Wager TD, Lopez R, Onyemekwu C, Kober H, et al. Cognitive reappraisal of emotion: a meta-analysis of human neuroimaging studies. Cereb Cortex 2014;24:2981-2990.

16. Kohn N, Eickhoff SB, Scheller M, Laird AR, Fox PT, Habel U. Neural network of cognitive emotion regulation -- an ALE meta-analysis and MACM analysis. Neuroimage 2014;87:345-355.

17. Dresler T, Hindi Attar C, Spitzer C, Lowe B, Deckert J, Buchel C, et al. Neural correlates of the emotional Stroop task in panic disorder patients: an event-related fMRI study. J Psychiatr Res 2012;46:1627-1634.

18. Shin LM, Whalen PJ, Pitman RK, Bush G, Macklin ML, Lasko NB, et al. An fMRI study of anterior cingulate function in posttraumatic stress disorder. Biol Psychiatry 2001;50:932-942.

19. Brown VM, Morey RA. Neural systems for cognitive and emotional processing in posttraumatic stress disorder. Front Psychol 2012;3:449.

20. Markowitsch HJ, Vandekerckhove MM, Lanfermann H, Russ MO. Engagement of lateral and medial prefrontal areas in the ecphory of sad and happy autobiographical memories. Cortex 2003;39:643-665.

21. Whalen PJ, Bush G, McNally RJ, Wilhelm S, McInerney SC, Jenike MA, et al. The emotional counting Stroop paradigm: a functional magnetic resonance imaging probe of the anterior cingulate affective division. Biol Psychiatry 1998;44:1219-1228.

22. Compton RJ, Banich MT, Mohanty A, Milham MP, Herrington J, Miller $\mathrm{GA}$, et al. Paying attention to emotion: an fMRI investigation of cognitive and emotional stroop tasks. Cogn Affect Behav Neurosci 2003;3:81-96.

23. Isenberg N, Silbersweig D, Engelien A, Emmerich S, Malavade K, Beattie B, et al. Linguistic threat activates the human amygdala. Proc Natl Acad Sci U S A 1999;96:10456-10459.

24. De Bellis MD, Keshavan MS. Sex differences in brain maturation in maltreatment-related pediatric posttraumatic stress disorder. Neurosci Biobehav Rev 2003;27:103-117.
25. Teicher MH, Dumont NL, Ito Y, Vaituzis C, Giedd JN, Andersen SL. Childhood neglect is associated with reduced corpus callosum area. Biol Psychiatry 2004;56:80-85.

26. Pechtel P, Lyons-Ruth K, Anderson CM, Teicher MH. Sensitive periods of amygdala development: the role of maltreatment in preadolescence. Neuroimage 2014;97:236-244.

27. Gee DG, Humphreys KL, Flannery J, Goff B, Telzer EH, Shapiro M, et al. A developmental shift from positive to negative connectivity in human amygdala-prefrontal circuitry. J Neurosci 2013;33:4584-4593.

28. Jeong B, Lee SW, Lee JS, Yoo JH, Kim KW, Cho S, et al. The psychometric properties of the Korean version of the verbal abuse questionnaire in university students. Psychiatry Investig 2015;12:190-196.

29. Spielberger CD, Gorsouh RL. Manual for the State-Trait Anxiety Inventory. Palo Alto, California: Consulting Psychologists Press; 1970.

30. Beck AT, Ward CH, Mendelson M, Mock J, Erbaugh J. An inventory for measuring depression. Arch Gen Psychiatry 1961;4:561-571.

31. Kim BR, Lee E, Kim HH, Park JY, Kang JI, An SK. Development of the Korean Affective Word List. J Korean Neuropsychiatr Assoc 2010;49: 468-479.

32. Sohn S, Park MS, Park JE, Sohn JH. Korean emotion vocabulary: extraction and categorization of feeling words. Korean J Sci Emot Sens 2012;15:105-120.

33. Kang SS, Oh KJ. Information processing bias for negative information in depressed group and socially anxious group. Korean J Clin Psychol 2003;22:17-32.

34. Kim H. Modern Korean Usage Frequency Survey. Seoul: The National Institute of The Korean Language; 2005.

35. Dresler T, Meriau K, Heekeren HR, van der Meer E. Emotional Stroop task: effect of word arousal and subject anxiety on emotional interference. Psychol Res 2009;73:364-371.

36. Jenkinson M, Beckmann CF, Behrens TE, Woolrich MW, Smith SM. FSL. Neuroimage 2012;62:782-790.

37. Rissman J, Gazzaley A, D’Esposito M. Measuring functional connectivity during distinct stages of a cognitive task. Neuroimage 2004;23:752-763.

38. Satterthwaite TD, Wolf DH, Loughead J, Ruparel K, Elliott MA, Hakonarson $\mathrm{H}$, et al. Impact of in-scanner head motion on multiple measures of functional connectivity: relevance for studies of neurodevelopment in youth. Neuroimage 2012;60:623-632.

39. Van Dijk KR, Sabuncu MR, Buckner RL. The influence of head motion on intrinsic functional connectivity MRI. Neuroimage 2012;59:431-438.

40. Shields A, Cicchetti D. Parental maltreatment and emotion dysregulation as risk factors for bullying and victimization in middle childhood. J Clin Child Psychol 2001;30:349-363.

41. Lisa H, David M. Sticks and Stones and Broken Bones: The Influence of Parental Verbal Abuse on Peer Related Victimization. West Crim Rev 2008;9:17-30.

42. Gallardo Perez M, Banos Rivera RM, Belloch Fuster A, Ruiperez Rodriguez MA. Attentional biases and vulnerability to depression. Span J Psychol 1999;2:11-19.

43. Phillips M, Ladouceur C, Drevets W. A neural model of voluntary and automatic emotion regulation: implications for understanding the pathophysiology and neurodevelopment of bipolar disorder. Mol Psychiatry 2008;13:829, 833-857.

44. Vytal K, Hamann S. Neuroimaging support for discrete neural correlates of basic emotions: a voxel-based meta-analysis. J Cogn Neurosci 2010;22:2864-2885.

45. Rive MM, van Rooijen G, Veltman DJ, Phillips ML, Schene AH, Ruhe HG. Neural correlates of dysfunctional emotion regulation in major depressive disorder. A systematic review of neuroimaging studies. Neurosci Biobehav Rev 2013;37:2529-2553.

46. Kalisch R. The functional neuroanatomy of reappraisal: time matters. Neurosci Biobehav Rev 2009;33:1215-1226.

47. Anderson MC, Ochsner KN, Kuhl B, Cooper J, Robertson E, Gabrieli SW, et al. Neural systems underlying the suppression of unwanted memories. Science 2004;303:232-235. 
48. Beauregard M, Levesque J, Bourgouin P. Neural correlates of conscious self-regulation of emotion. J Neurosci 2001;21:RC165.

49. Greenberg DL, Rice HJ, Cooper JJ, Cabeza R, Rubin DC, Labar KS. Co-activation of the amygdala, hippocampus and inferior frontal gyrus during autobiographical memory retrieval. Neuropsychologia 2005;43:659-674.

50. Tupak SV, Dresler T, Guhn A, Ehlis AC, Fallgatter AJ, Pauli P, et al. Implicit emotion regulation in the presence of threat: neural and autonomic correlates. Neuroimage 2014;85 Pt 1:372-379.

51. Monk CS, Nelson EE, McClure EB, Mogg K, Bradley BP, Leibenluft E, et al. Ventrolateral prefrontal cortex activation and attentional bias in response to angry faces in adolescents with generalized anxiety disorder. Am J Psychiatry 2006;163:1091-1097.

52. van Harmelen AL, van Tol MJ, Demenescu LR, van der Wee NJ, Veltman DJ, Aleman A, et al. Enhanced amygdala reactivity to emotional faces in adults reporting childhood emotional maltreatment. Soc Cogn Affect Neurosci 2013;8:362-369.

53. Dannlowski U, Kugel H, Huber F, Stuhrmann A, Redlich R, Grotegerd $\mathrm{D}$, et al. Childhood maltreatment is associated with an automatic negative emotion processing bias in the amygdala. Human Brain Mapp 2013;34:2899-2909.

54. Dannlowski U, Stuhrmann A, Beutelmann V, Zwanzger P, Lenzen T, Grotegerd D, et al. Limbic scars: long-term consequences of childhood maltreatment revealed by functional and structural magnetic resonance imaging. Biol Psychiatry 2012;71:286-293.

55. Grant MM, Cannistraci C, Hollon SD, Gore J, Shelton R. Childhood trauma history differentiates amygdala response to sad faces within MDD. J Psychiatr Res 2011;45:886-895.

56. Tottenham N, Hare TA, Millner A, Gilhooly T, Zevin JD, Casey BJ. Elevated amygdala response to faces following early deprivation. Dev Sci 2011;14:190-204.

57. Maheu FS, Dozier M, Guyer AE, Mandell D, Peloso E, Poeth K, et al. A preliminary study of medial temporal lobe function in youths with a history of caregiver deprivation and emotional neglect. Cogn Affect Behav Neurosci 2010;10:34-49.
58. Thomaes K, Dorrepaal E, Draijer N, de Ruiter MB, Elzinga BM, Sjoerds Z, et al. Increased anterior cingulate cortex and hippocampus activation in Complex PTSD during encoding of negative words. Soc Cogn Affect Neurosci 2013;8:190-200.

59. Yeterian EH, Pandya DN, Tomaiuolo F, Petrides M. The cortical connectivity of the prefrontal cortex in the monkey brain. Cortex 2012;48:58-81.

60. Roberts AC, Tomic DL, Parkinson CH, Roeling TA, Cutter DJ, Robbins TW, et al. Forebrain connectivity of the prefrontal cortex in the marmoset monkey (Callithrix jacchus): an anterograde and retrograde tract-tracing study. J Comp Neurol 2007;502:86-112.

61. Barbas H, Blatt GJ. Topographically specific hippocampal projections target functionally distinct prefrontal areas in the rhesus monkey. Hippocampus 1995;5:511-533.

62. Schacter DL, Wagner AD. Medial temporal lobe activations in fMRI and PET studies of episodic encoding and retrieval. Hippocampus 1999;9:7-24.

63. Strange BA, Fletcher PC, Henson RN, Friston KJ, Dolan RJ. Segregating the functions of human hippocampus. Proc Natl Acad Sci U S A 1999;96:4034-4039.

64. Lepage M, Habib R, Tulving E. Hippocampal PET activations of memory encoding and retrieval: the HIPER model. Hippocampus 1998; 8:313-322.

65. Papanicolaou AC, Simos PG, Castillo EM, Breier JI, Katz JS, Wright AA. The hippocampus and memory of verbal and pictorial material. Learn Mem 2002;9:99-104.

66. Kelley WM, Miezin FM, McDermott KB, Buckner RL, Raichle ME, Cohen NJ, et al. Hemispheric specialization in human dorsal frontal cortex and medial temporal lobe for verbal and nonverbal memory encoding. Neuron 1998;20:927-936.

67. Badre D, Poldrack RA, Pare-Blagoev EJ, Insler RZ, Wagner AD. Dissociable controlled retrieval and generalized selection mechanisms in ventrolateral prefrontal cortex. Neuron 2005;47:907-918.

68. Barredo J, Oztekin I, Badre D. Ventral fronto-temporal pathway supporting cognitive control of episodic memory retrieval. Cereb Cortex 2015;25:1004-1019. 